\section{Tofacitinib is not a biologic}

\section{Amy S. Marren}

Pfizer Inc

As the Global Medical Affairs Gastroenterology Lead for tofacitinib at Pfizer, I read with great interest the review by Dr Ungar and Dr Kopylov entitled "Advances in the development of new biologics in inflammatory bowel disease (IBD)" [1].

In reading the article, I identified a number of inaccuracies concerning tofacitinib, which I thought should be brought to the attention of the authors and your readers. Throughout the article tofacitinib is described as being a "biologic". Although it may be appropriate to compare the efficacy and safety of tofacitinib to the existing biologic therapies used in IBD, tofacitinib is a synthetic small molecule JAK inhibitor and not a biologic agent.

Tofacitinib was introduced along with descriptions of the biologics, including anti-integrins and ustekinumab. It would be more accurate if tofacitinib was split from these biologic agents and instead described in a separate sentence, since antiintegrins and ustekinumab are biologics and tofacitinib is not.

In relation to safety events, in place of the current "The adverse effect profile for tofacitinib appears to be similar to other biologics...", it would be more accurate to state that "The adverse effect profile for tofacitinib appears to be similar to that of biologics..., thus separating tofacitinib from the biologic drug class.

With respect to the discussion around immunogenicity and tofacitinib, because tofacitinib is a synthetic small molecule JAK inhibitor and not a biologic agent we would not expect there to be any immunogenicity. Hence, no specific studies of the immunogenicity of tofacitinib have been performed or are planned at present.

Finally, we are nearing completion of a phase 3 development program of tofacitinib in ulcerative colitis $[2,3]$ and we look forward to sharing the results of these studies with the gastroenterological community in the future.

\section{References}

1. Ungar B, Kopylov U. Advances in the development of new biologics in inflammatory bowel disease. Ann Gastroenterol 2016;29:243.

2. ClinicalTrials.gov. A study of oral CP-690,550 as a maintenance therapy for ulcerative colitis (OCTAVE). Available at: https:// clinicaltrials.gov/ct2/show/NCT01458574. Accessed August 26, 2016.

3. ClinicalTrials.gov. Long-term study of CP-690,550 in subjects with ulcerative colitis (OCTAVE). Available at: https://clinicaltrials.gov/ ct2/show/NCT01470612. Accessed August 26, 2016.

Medical Lead/Team Leader Gastroenterology, Global Medical Affairs, Pfizer Inc, PA, USA

Conflict of Interest: Amy S. Marren is an employee and shareholder of Pfizer Inc

Correspondence to: Amy S. Marren, Medical Lead/Team Leader Gastroenterology, Global Medical Affairs, Pfizer Inc, 500 Arcola Rd, Collegeville, PA 19426, USA, e-mail: Amy.Marren@pfizer.com

Received 2 September 2016; accepted 8 September 2016; published online 7 October 2016

DOI: https://doi.org/10.20524/aog.2016.0094 